\title{
Optimal Operation of GENCOs in Competitive Electricity Markets
}

\author{
Olamide A. Oriola, Nurul Chowdhury \\ University of Saskatchewan \\ 57 Campus Drive, Saskatoon, Canada \\ olamide.oriola@usask.ca; nurul.chowdhury@usask.ca
}

\begin{abstract}
In a deregulated power system, power generators submit offers to sell energy and operating reserve in the electricity market. The market can be described as an oligopoly due to certain characteristics such as a restricted number of producers. A sealed bid auction is the usual practice with competing generators having no information on rivals' bids. This paper presents a technique for power producers to make security-constrained offers in different electricity markets considering incomplete market information and uncertainty in load forecast. The methodology employed is based on forecasting and optimization. Electricity market clearing price at each interval is predicted using the double seasonal Holt-Winters method and used in the optimization problem of profit maximization to estimate maximum benefit at the interval. Economic dispatch of committed generating units is also evaluated using a dynamic programming procedure to minimize production cost. A numerical example serves to illustrate the proposed approach as it is applied to a practical system. Results indicate that a generator can make adequate short-term analysis on market behavior and maximize its benefits for the period based on available historical data on market operation.
\end{abstract}

Keywords: Power system, Economic dispatch, Electricity market, Forecasting, Uncertainty, Market clearing price.

\section{Introduction}

In a deregulated system, an Independent System Operator (ISO) manages the operations of the power grid in a region. It ensures an economic operation by balancing power producers' supply offers and consumers' bids in energy markets at the lowest possible price, determining the market clearing price (MCP). MCP is the price at the point of equilibrium where there is a balance between supply and demand for energy. All generators with offer prices below or equal the MCP are scheduled to supply at the market interval and are paid the MCP regardless of what their offer price was. This single price approach encourages generating companies (GENCOs) to make offers at least possible cost and limits market manipulation. The sealed bid auction is widely used in ISO operated power grids. Bids are submitted simultaneously within a timeframe and are confidential, thus a generator has no information on rivals' bids.

The day-ahead and spot markets are types of energy market for the wholesale trade of electricity. A day-ahead market (DAM) is a forward market for energy needed at each hour of the following day. The market opens few days prior to the trade day and closes a day before the trade day. A spot market (SM) is a real-time market to maintain continuous balance between supply and demand of energy on the trade day. The market is an avenue for utilities to procure additional energy needed to meet demand when real-time demand is more than forecasts. Auction process in the SM is similar to that of the DAM but with a shorter timeline. The shorter timeline is a contributing factor to the high energy price feature of the SM.

In recent years, there has been significant volume of research on market structure and bidding strategies for generators in competitive electricity markets. Popular methods for electricity market modelling can be grouped into equilibrium models, simulation models, and optimization models [1]. Equilibrium models analyze the competitive market considering behavior and strategy of all GENCOs. The method applies techniques from game theory to simultaneously maximize profit of each generator. Equilibrium models are based on the principle of Nash equilibrium [2]. The Cournot model [3] is an example of equilibrium models where competing generators base competition on production quantity. Another example of equilibrium models is the supply function equilibrium (SFE) approach introduced in [4]. The approach is a better strategy for modelling uncertainty in electricity markets by relating quantity and price when compared to the Cournot model as seen in its application in [5]-[8]. The output of the SFE model is a bid curve stating price and corresponding quantity.

Optimization models [9]-[14] presents analysis of strategic bidding by a single firm in the electricity market where the objective is to maximize profit subject to some technical and economic constraints. All of these techniques assume knowledge of rivals' bid coefficients or access to sufficient data on bidding history to estimate rivals' behavior. A framework 
for strategic bidding by power producers was developed in [9]. The optimal bidding problem was solved using Monte-Carlo simulation and an optimization-based technique.

This paper aims to examine a technique for GENCOs to maximize daily benefits from their operation in the day-ahead and spot electricity markets while maintaining system security. The approach analyzes the market from a single GENCO's point of view, maximizing individual profit. With little information on auction process and bidding history, this paper explores building a GENCO's optimal offers by estimating MCP for each hour of the trade day in a DAM, extending this to the SM and incorporating possible risk with uncertainty in demand while acknowledging high prices feature of the SM. The proposed approach models the general problem of obtaining maximum profits from the electricity markets as a constrained optimization problem. The first step to solving the GENCO's problem is to estimate next day's prices in the electricity markets. Double seasonal Holt-Winters (DSHW) method, a time series model based on exponential smoothing, is introduced for short-term electricity price forecasting. With the predicted prices, the objective function of the optimization problem is solved for profit-maximizing offer quantity using the golden section search optimization (GSSO). For cost minimization, an economic distribution of load (offer quantity in this case) is done among committed units. This process of economic dispatch ensures that the GENCO is operating at the least incremental running cost. This is an important step in solving the optimization problem for proper estimation of expected daily market benefits and economic operation of units. Economic dispatch of units which is solved using the dynamic programming (DP) procedure would make the last step of the proposed approach to profit-maximization. The analysis examines simultaneous participation in DAM and SM, obtaining optimal benefits from both markets. This is a short-term analysis, not more than 14 days prior to the trade day.

\section{Problem Formulation}

The objective of a GENCO is to maximize benefit from the electricity market for each hour of the trade day while minimizing cost. Cost associated with power generation has two components: fixed cost and variable cost. A GENCO's variable costs consists of fuel cost and operations and maintenance cost. The model assumed in this paper relates to GENCOs with thermal plants, thus fuel cost is to be minimized. If the valve point effect of a thermal power plant is ignored, the fuel cost can be described as a smooth function defined by polynomial functions [15]. Quadratic fuel cost function for thermal power plants is given as:

$$
F C_{i}\left(P_{i}\right)=a_{i} P_{i}^{2}+b_{i} P_{i}+c_{i} \quad i=1,2, \cdots, M
$$

where $F C_{i}$ is the fuel cost function $(\$ / H r)$ of the ith generating unit, $a_{i}, b_{i}$ and $c_{i}$ are the cost coefficients, and $P_{i}$ is the power output in MW of the ith thermal unit. $M$ is the total number of thermal generating units present.

The problem of daily benefit for 24 separate hourly auctions is described as:

$$
\begin{gathered}
\text { maximize } p(Q(h)) \\
p(Q(h))=R(h) * Q(h)-C(Q(h)) \quad h=1,2, \cdots, 24 \\
Q(h)=\sum_{i=1}^{M} P_{i}(h) \\
C(Q(h))=\sum_{i=1}^{M} F C_{i}(h)
\end{gathered}
$$

Subject to: $P_{i}^{\min } \leq P_{i}(h) \leq P_{i}^{\max }$

where $Q(h)$ is the production quantity at hour $h, R(h)$ is the forecast market clearing price at hour $h, C(Q(h))$ is the total cost of producing quantity $Q(h)$ and $p(Q(h))$ is the profit from producing quantity $Q(h)$. 
Equation (3) presents hourly profit as revenue minus production cost. For optimal benefit, offers to be submitted for the trade day should be decided in a way as to minimize risk of not being selected to supply. Equation (3) has two variables: $\mathrm{MCP}$ and production quantity. Estimating the MCP would give an insight into the quantity that can be offered at a given price. The proposed approach to solving the GENCO's optimal benefit problem is presented by the simplified flow diagram in Fig. 1. MCP forecast is obtained utilizing Double Seasonal Holt-Winters (DSHW) model. Equation (4) presents the offer quantity $Q(h)$ to be the summation of output from each generating unit running at hour $h . Q(h)$ is computed using Golden Section Search Optimization (GSSO). Equation (5) relates the total cost of producing quantity $Q(h)$ as the summation of running cost of all generating unit operating at the hour, while the inequality constraint in (6) is the generating unit's output constraint.

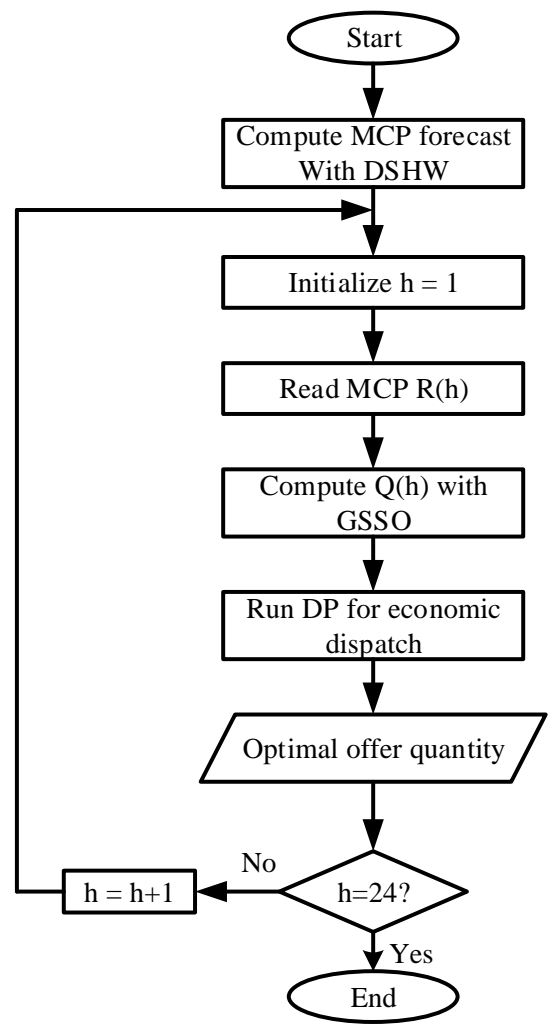

Fig. 1: Flow diagram of proposed approach.

When ISO decides on dispatch schedules for the trade day, GENCOs scheduled are under obligation to make allocated generation available and supply as stated by dispatch instructions. To plan for unforeseen events such as sudden loss of generation that could cause a GENCO to fall short of the scheduled supply, a probabilistic method of risk assessment is incorporated in the decision process for making offers. Spinning reserve (SR) is determined from a capacity outage probability evaluation and allocated as reserve capacity to accommodate contingencies.

A GENCO solves its economic dispatch problem when making offers in an electricity market in a manner similar to that of a regulated power system. The general idea behind economic dispatch is to have the unit with the lowest marginal cost dispatched first. For a GENCO with $M$ number of generating units, the hourly economic dispatch problem can be explained as:

$$
\text { minimize } \sum_{i=1}^{M} F C_{i}\left(P_{i}(h)\right)
$$




$$
\begin{aligned}
& \text { subject to: } D(h)-\sum_{i=1}^{M} P_{i}(h)=0 \\
& Q(h)+S R(h) \leq U C(h) \\
& \text { and (6) }
\end{aligned}
$$

$F C_{i}\left(P_{i}(h)\right)$ is the fuel cost of unit $i$ at hour $h$. Equation (8) represents the power balance which states that the total generation at hour $h$ should be equal to demand, $D(h)$. Demand in this context is the estimated profit-maximizing quantity, $Q(h)$. Constraint in (9) describes that the sum of the offer quantity, $Q(h)$ and the spinning reserve, $S R(h)$ should be less than or equal to $U C(h)$, the total capacity of the units committed at hour $h$.

\subsection{Double Seasonal Holt-Winters Model}

The standard Holt-Winters model introduced in [16] is based on triple exponential smoothing for forecasting seasonal time series. Extending standard Holt-Winters' method of forecasting seasonal time series with one seasonal pattern, [17] described a modification of the method to accommodate time series with dual seasonal pattern. Equations presenting the double seasonal Holt-Winters method are as follows:

$$
\begin{gathered}
\mathrm{L}_{\mathrm{t}}=\alpha\left(\mathrm{X}_{\mathrm{t}} /\left(\mathrm{DS}_{\mathrm{t}-\mathrm{p} 1} \mathrm{WS}_{\mathrm{t}-\mathrm{p} 2}\right)\right)+(1-\alpha)\left(\mathrm{L}_{\mathrm{t}-1}+\mathrm{T}_{\mathrm{t}-1}\right) \\
\mathrm{T}_{\mathrm{t}}=\gamma\left(\mathrm{L}_{\mathrm{t}}-\mathrm{L}_{t-1}\right)+(1-\gamma) \mathrm{T}_{\mathrm{t}-1} \\
\mathrm{DS}_{\mathrm{t}}=\delta\left(\mathrm{X}_{\mathrm{t}} /\left(\mathrm{L}_{\mathrm{t}} \mathrm{WS}_{\mathrm{t}-\mathrm{p} 2}\right)\right)+(1-\delta) \mathrm{DS}_{\mathrm{t}-\mathrm{p} 1} \\
\mathrm{WS}_{\mathrm{t}}=\omega\left(\mathrm{X}_{\mathrm{t}} /\left(\mathrm{L}_{\mathrm{t}} \mathrm{DS}_{\mathrm{t}-\mathrm{p} 1}\right)\right)+(1-\omega) \mathrm{WS}_{\mathrm{t}-\mathrm{p} 2} \\
\widehat{\mathrm{X}}_{\mathrm{t}}(\mathrm{k})=\left(\mathrm{L}_{\mathrm{t}}+\mathrm{kT}_{\mathrm{t}}\right) \mathrm{DS}_{\mathrm{t}-\mathrm{p} 1+\mathrm{k}} \mathrm{WS}_{\mathrm{t}-\mathrm{p} 2+\mathrm{k}}
\end{gathered}
$$

where $t$ is an index denoting a time period, $X_{t}$ is the observed value, $L_{t}$ is the level and $T_{t}$ the trend. $\mathrm{DS}_{t}$ and $\mathrm{WS}_{\mathrm{t}}$ are the first and second seasonality, both with period $\mathrm{p} 1$ and $\mathrm{p} 2 . \alpha, \gamma, \delta$ and $\omega$ are smoothing parameters in the range $[0,1] . \widehat{\mathrm{X}}_{\mathrm{t}}(\mathrm{k})$ is the forecast for $\mathrm{k}$ hours ahead. The method estimates local slope (trend) by smoothing successive differences between levels. Applying it to hourly MCP forecasting in electricity markets, $\mathrm{DS}_{\mathrm{t}}$ and $\mathrm{WS}_{\mathrm{t}}$ would represent the daily and weekly seasonality respectively. $\mathrm{p} 1$ is thus set to 24 and p2 to 168. Historical day-ahead auction MCPs from Nord Pool is used to test the model. The actual and forecast values for the week January $1^{\text {st }}$ to January $7^{\text {th }}, 2017$ are shown in Fig. 2.

\section{Numerical Example}

A test system consisting of twelve thermal generating units of different sizes [18] is used to demonstrate the proposed approach. The forecast load of January 5, 2017 of Ontario Independent System Operator were utilized as the forecast load of a typical trade day. Total system maximum and minimum capacity are $3450 \mathrm{MW}$ and $1166 \mathrm{MW}$ respectively. The priority order, fuel cost coefficients and generating limits are given in Table 1. The utility's commitment is based on maximizing benefit with the maximum possible generating capacity. 


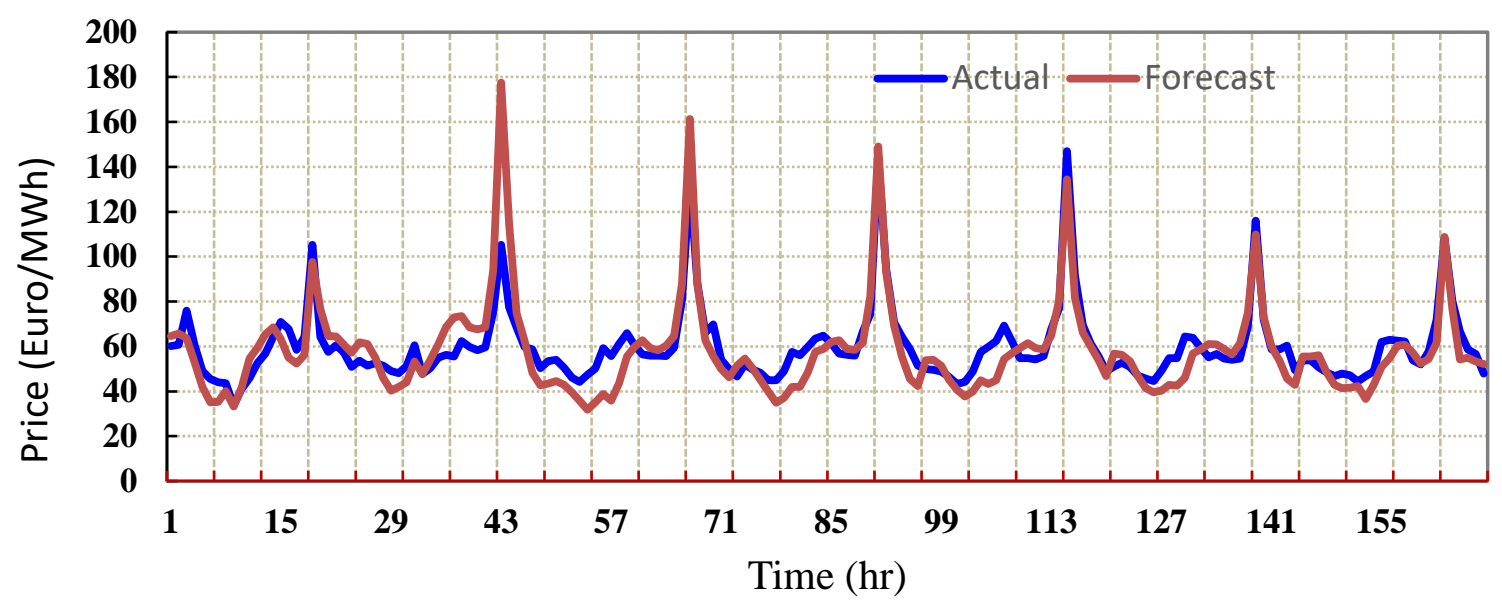

Fig. 2: One-week Nord Pool real and simulated day-ahead auction MCP.

Table 1: Generation Data.

\begin{tabular}{cccccc}
\hline $\begin{array}{c}\text { Loading } \\
\text { Order }\end{array}$ & $\mathrm{a}_{\mathrm{i}}$ & $\mathrm{b}_{\mathrm{i}}$ & $\mathrm{c}_{\mathrm{i}}$ & $\begin{array}{c}\mathrm{P}_{\max } \\
(\mathrm{MW})\end{array}$ & $\begin{array}{c}\mathrm{P}_{\min } \\
(\mathrm{MW})\end{array}$ \\
\hline 1 & 0.03073 & 8.336 & 170.44 & 80 & 40 \\
2 & 0.02028 & 7.0706 & 309.54 & 120 & 60 \\
3 & 0.01142 & 8.0543 & 222.33 & 140 & 68 \\
4 & 0.00942 & 8.1817 & 369.03 & 190 & 80 \\
5 & 0.00357 & 8.0323 & 287.71 & 300 & 110 \\
6 & 0.25098 & 13.052 & 1207.8 & 70 & 20 \\
7 & 0.00605 & 12.908 & 722.82 & 300 & 130 \\
8 & 0.00313 & 7.9691 & 647.85 & 500 & 220 \\
9 & 0.00515 & 12.986 & 635.2 & 375 & 94 \\
10 & 0.00569 & 12.796 & 654.69 & 375 & 94 \\
11 & 0.00708 & 9.1575 & 1728.3 & 500 & 125 \\
12 & 0.00421 & 12.501 & 913.4 & 500 & 125 \\
\hline
\end{tabular}

\subsection{Bidding in Day-ahead Market}

As previously described, the first step is to estimate hourly MCPs for the trade day. With the market opening about 7 to 10 days prior to trade day and closing a day before the trade day, short-term estimated MCPs are marked as offer price for the maximum benefit and used in the optimization algorithm to determine offer quantity.

For predicted prices shown in Fig. 3, (2) and (7) are solved for profit-maximizing offer quantity while considering system security. Equation (2) is solved using the golden section search algorithm in each one-dimensional search to get $Q(h)$ with unit's generating limits as search boundary. $S R(h)$ is estimated and $Q(h)$ adjusted if necessary when solving (7) to keep reserve requirements.

Market returns are seen at lowest between hours 5 and 10 and hour 14 where price is low. Increasing offer quantity above what is estimated to be scheduled at that price would result in GENCO operating at a loss. Keeping SR would translate to a decrease in maximum offer quantity possible and an increase in operating cost, reducing market benefits. However, maintaining reliability is important in system operation. Considering a 3\% uncertainty in load forecast described by a 5-step approximation of the normal distribution, Fig. 6 shows aggregate benefits from the DAM obtained from weighted sum of benefit with five estimated MCPs based on each of the load profiles. The uncertainty is in the range $[\mu-2 \sigma, \mu+2 \sigma]$ with $\mu$ as 
the forecast mean and $\sigma$ the standard deviation. Output of the scenario before uncertainty in load forecast was considered and that of aggregate from load forecast uncertainty (LFU) which seem to be within a close range is a result of prices used in the optimization process. At hour 1, benefit shown by Fig. 5 is $\$ 24965.29$ and $\$ 26259.05$ from Fig. 6 . Hour 7 has a wider difference with $\$ 1976.94$ before uncertainty and \$4534.86 with LFU.

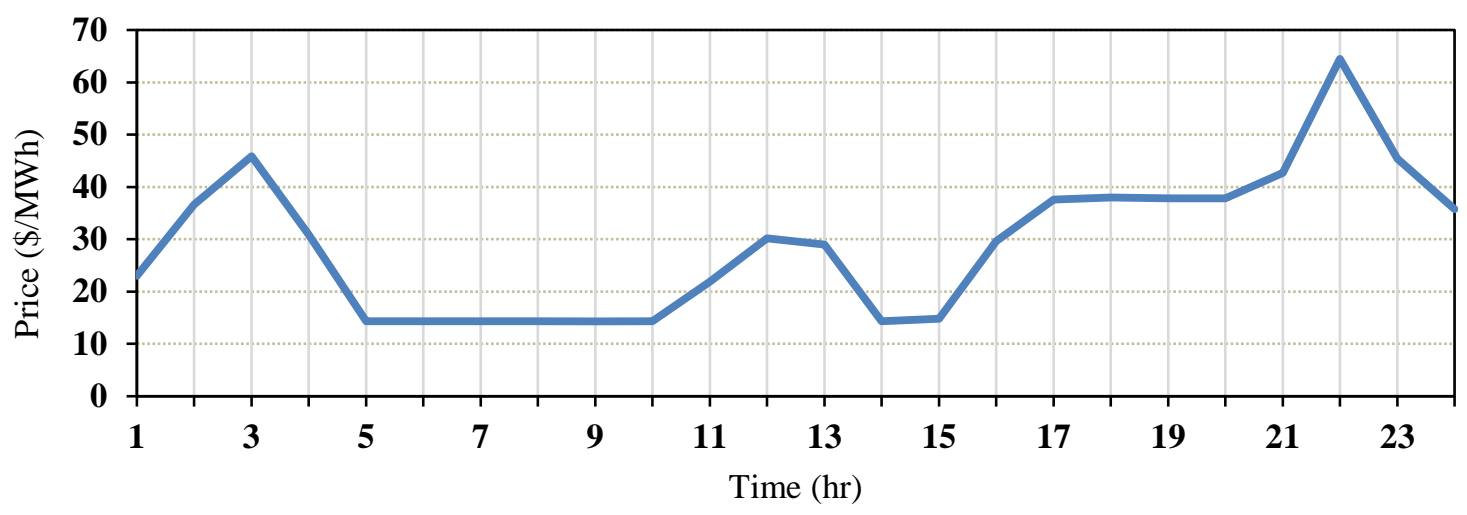

Fig. 3: Estimated DAM prices for the trade day.

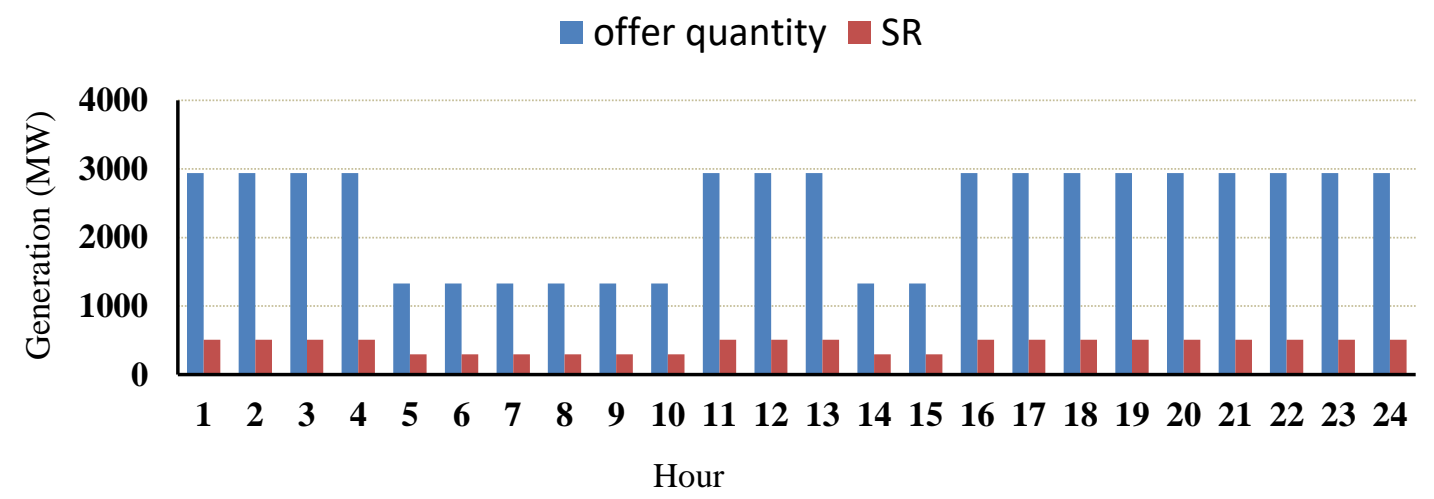

Fig. 4: Hourly offer quantity and reserve capacity.

\subsection{Bidding in Day-ahead and Spot Markets}

Transaction for power supply to satisfy real-time increase in market demand which could not have been scheduled in the DAM is done in the SM. Energy procured from this market is at a different price; the spot market price, which can be very high when compared to the DAM prices. Fig. 7 shows hourly aggregate market benefits from the SM if the GENCO's participation in DAM auction takes precedence over its offers in the SM. Hours with profit of $\$ 0$ stems from zero revenue. This is as a result of the GENCO not participating in the SM at these periods as all its available capacity has been committed in the DAM auction. SR requirement is maintained and actually increases with commitment in the SM. 


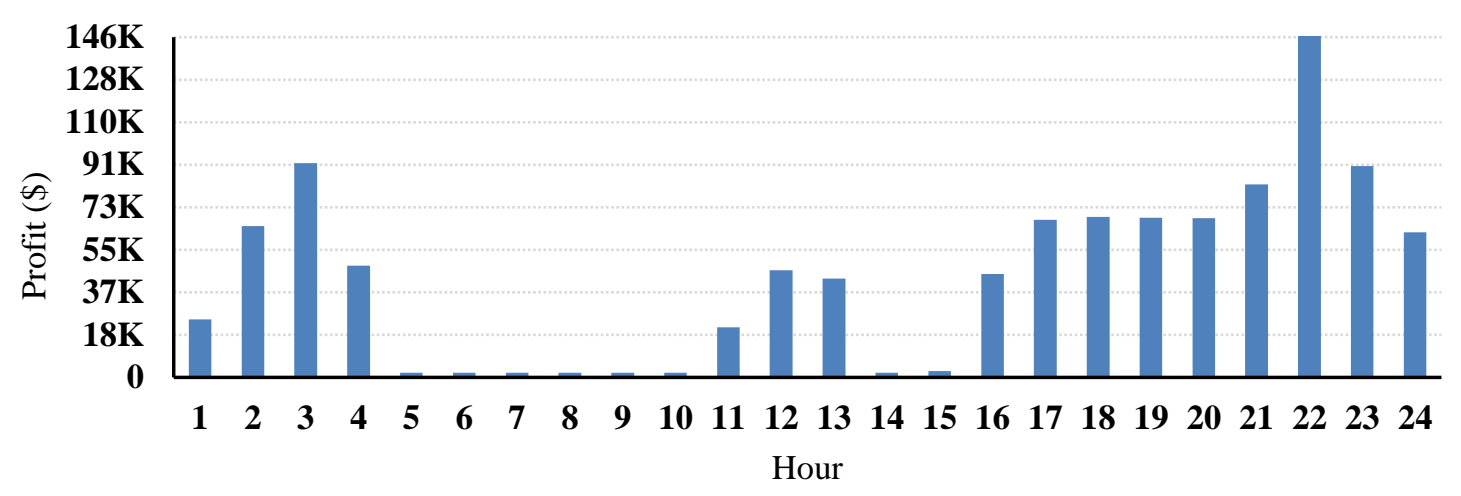

Fig. 5: GENCO's benefits from DAM auction process considering reserve capacity for system security.

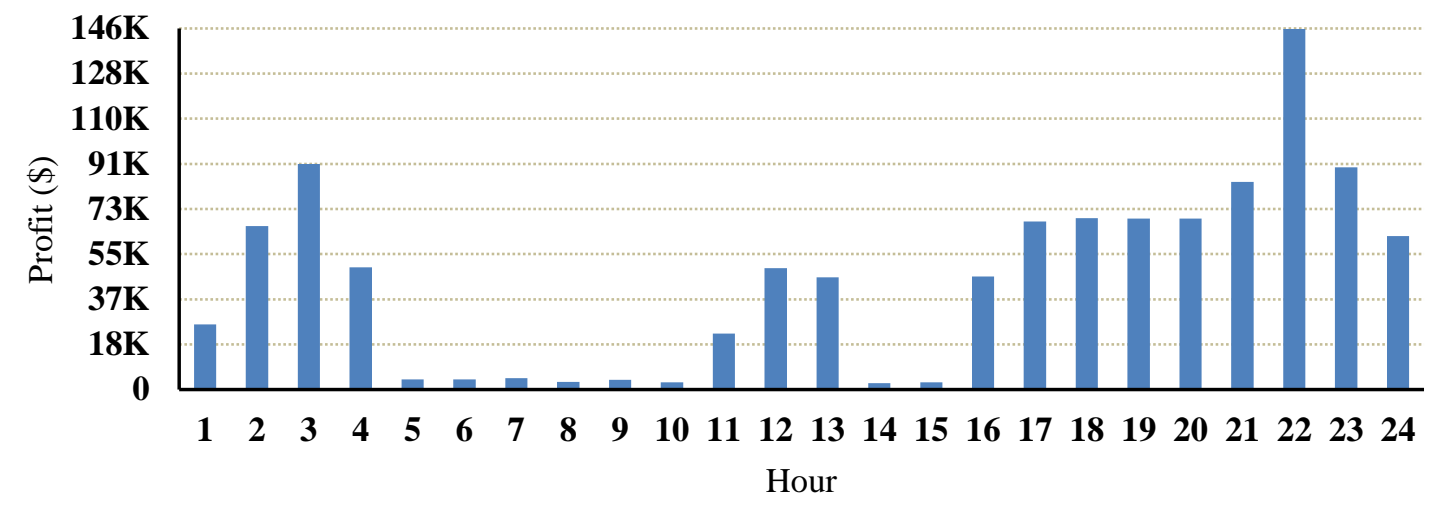

Fig. 6: GENCO’s aggregate benefits from DAM auction process.

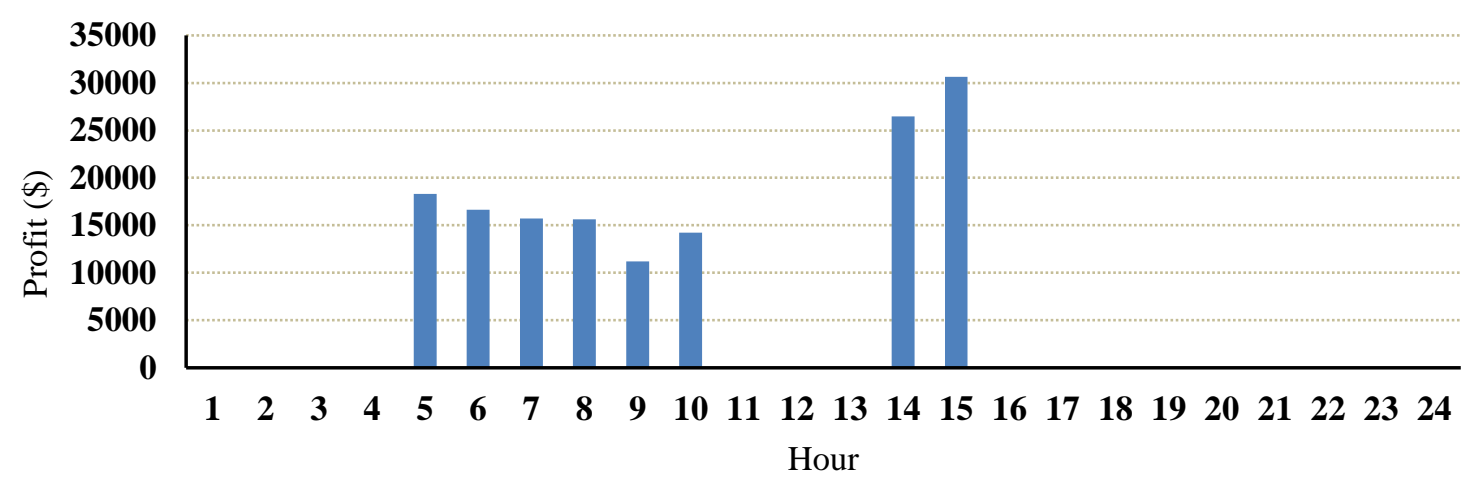

Fig. 7: GENCO's aggregate benefits from SM.

Quantity of energy transacted in a SM is lower when compared with energy traded in DAM since the former is meant to make up for differences present. Also, energy is only required from a SM if real-time demand is higher than forecast, thus the increasing step deviation half $(+\sigma)$ of the LFU modelling is considered. The scenario with results shown in Fig. 7 commits capacity left from bidding in the DAM in the SM. However, due to efficient forecasting methods, difference in real-time demand and forecast is usually not very large. With high prices as a feature of the SM, an allocation of the GENCO's generation capacity to both the DAM and the SM is considered. To participate in both markets concurrently at every hour, 95\% of generation is committed in the DAM auction at periods where commitment was previously maximum and 5\% in the SM for an uncertainty of $\mu+\sigma$ in demand. For a deviation of $\mu+2 \sigma, 90 \%$ and $10 \%$ of generation are committed in the DAM 
and SM respectively. Fig. 8 shows hourly aggregate market benefits from the SM. The GENCO has a commitment for the SM at every hour unlike what can be observed in Fig. 7. Although the GENCO's benefit is lower with percentage allocation considered, committing very large quantity of generation in the SM is rather optimistic and can result in a loss for the GENCO.

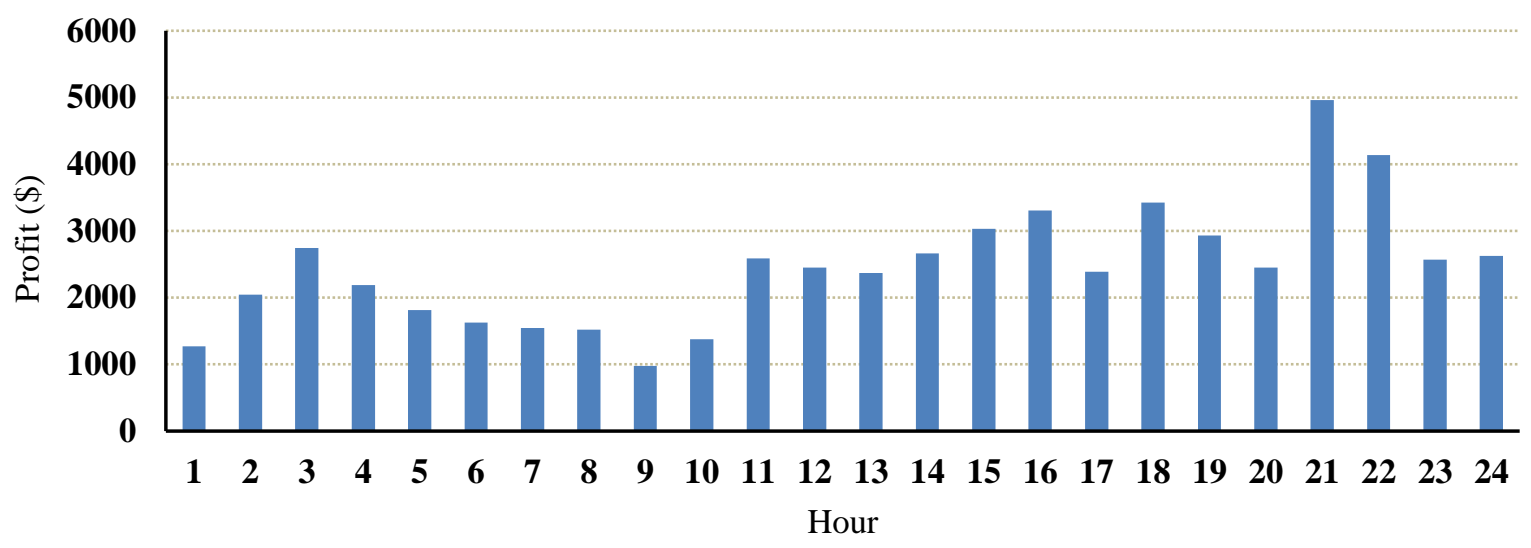

Fig. 8: GENCO’s aggregate benefits from SM with percentage allocation.

The day profit from the DAM auction decreases when compared with previous scenarios since generation quantity committed is lesser. However, overall day benefits from both markets is an improvement on this due to high energy prices feature of the SM. Estimated benefits at the end of the trade day from participating in both day-ahead and spot electricity markets are presented in Fig. 9. SR for GENCO's system security is kept as a requirement for all periods and in both markets with capacity on reserve increasing with increase in load level. The initial case of aggregate day profit represents GENCO trading only in DAM considering LFU (Fig. 6). LFU is thus considered from two aspects. First is variability in market demand for DAM auction with GENCO making commitments only in the DAM. The LFU is considered in the decision process for supply offers in the DAM. Second is participation in SM as a result of uncertainty in demand. In this situation the commitment for DAM remain as decided but decision process includes SM at $+\sigma$ uncertainty.

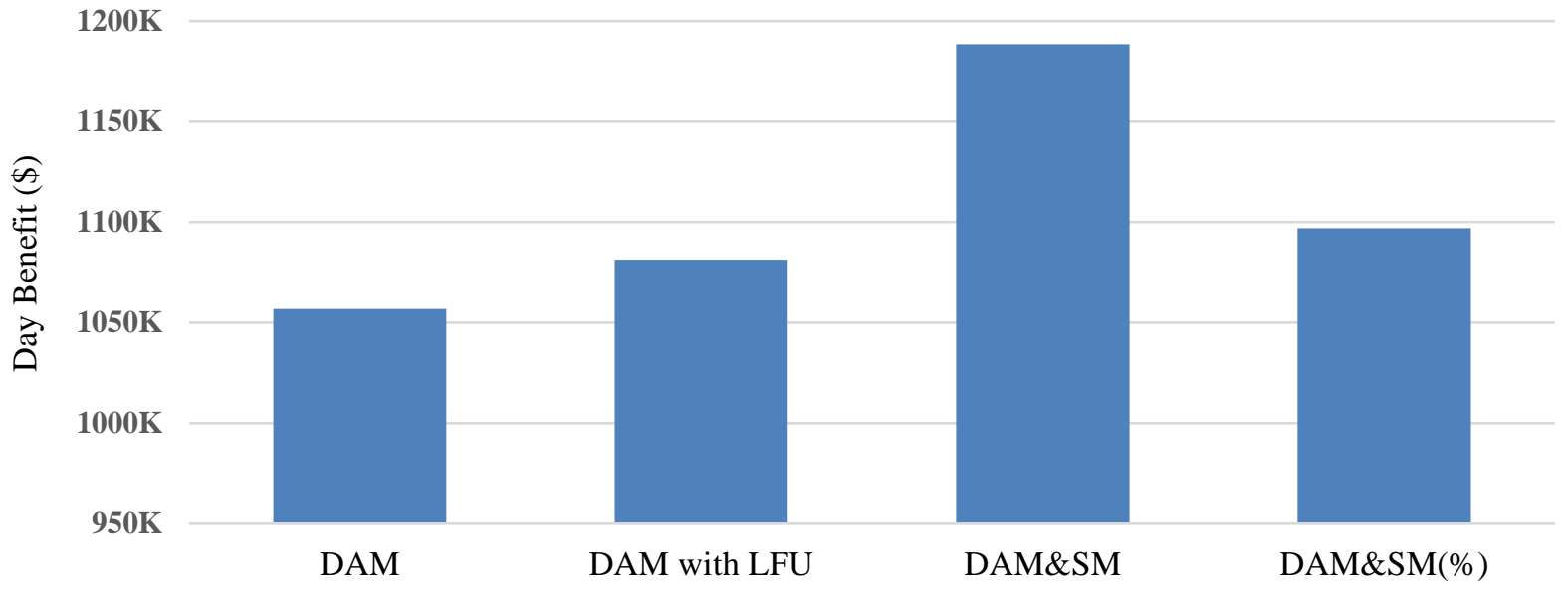

Fig. 9: GENCO's estimated benefits at the end of trade day.

\section{Conclusion}

A method to solve a single GENCO's problem of making profit-maximizing offers in the day-ahead and spot electricity markets has been presented. The technique involve short-term price prediction, maximization of expected profit and cost minimization taking note of necessary reserve requirement for system security. An example of a GENCO with twelve thermal 
units has been used to demonstrate the method and results provide insights into strategies that can be employed by GENCOs for maximum benefits from their participation in electricity markets. Notwithstanding the governing market rules which vary with regions, method presented would prove useful in analyzing simultaneous operation of GENCOs in the DAM and SM which are the markets for the bulk trade of electricity.

\section{References}

[1] M. Ventosa, Á. Baíllo, A. Ramos, and M. Rivier, "Electricity market modeling trends," Energy Policy, vol. 33, no. 7, pp. 897-913, 2005.

[2] H. Peters, Game theory: A Multi-Leveled Approach, 2nd ed. Springer Heidelberg, 2015, pp. 2-44.

[3] A. Maiorano, Y. H. Song, and M. Trovato, "Modelling and analysis of electricity markets," in Operation of Marketoriented Power Systems, Y. H. Song and X. F. Wang, Eds. Springer-Verlag, 2003, pp. 13-46.

[4] P. D. Klemperer and M. A. Meyer, "Supply function equilibria in oligopoly under uncertainty," Econometrica, vol. 57, no. 6, pp. 1243-1277, 1989.

[5] R. J. Green and D. M. Newbery, “Competition in the British electricity spot market,” J. Polit. Econ., vol. 100, no. 5, pp. 929-953, 1992.

[6] R. Green, "Increasing competition in the British electricity spot market," J. Ind. Econ., vol. 44, no. 2, pp. 205-216, 1996.

[7] R. Baldick, R. Grant, and E. Kahn, "Theory and application of linear supply function equilibrium in electricity markets," J. Regul. Econ., vol. 25, no. 2, pp. 143-167, 2004.

[8] S. Y. Al-Agtash, "Supply curve bidding of electricity in constrained power networks," Energy, vol. 35, no. 7, pp. 2886-2892, 2010.

[9] F. Wen and A. K. David, "Optimal bidding strategies and modeling of imperfect information among competitive generators," IEEE Trans. Power Syst., vol. 16, no. 1, pp. 15-21, 2001.

[10] F. S. Wen and A. K. David, "Strategic bidding for electricity supply in a day-ahead energy market," Electr. Power Syst. Res., vol. 59, no. 3, pp. 197-206, 2001.

[11] V. P. Gountis and A. G. Bakirtzis, "Bidding strategies for electricity producers in a competitive electricity marketplace," IEEE Trans. Power Syst., vol. 19, no. 1, pp. 356-365, 2004.

[12] J. V. Kumar and D. M. V. Kumar, "Optimal bidding strategy in a competitive electricity market using differential evolution," in Annu. IEEE India Conf., Hyderabad, India, 2011, pp. 1-5.

[13] X. Guan, Y. Ho, and F. Lai, "An ordinal optimization-based bidding strategy for electric power suppliers in the daily energy market," IEEE Trans. Power Syst., vol. 16, no. 4, pp. 788-797, 2001.

[14] D. Singh and A. Kumar, "Bidding strategy for competitive electricity market by using optimization technique (PSO \&APSO)," Int. J. Eng. Res. Technol., vol. 3, no. 5, pp. 1541-1548, 2014.

[15] Y. Sönmez, "Estimation of fuel cost curve parameters for thermal power plants using the ABC algorithm," Turkish J. Electr. Eng. Comput. Sci., vol. 21, pp. 1827-1841, 2013.

[16] P. R. Winters, "Forecasting sales by exponentially weighted moving averages," Manage. Sci., vol. 6, no. 3, pp. 324342, 1960.

[17] J. W. Taylor, "Short-term electricity demand forecasting using double seasonal exponential smoothing," J. Oper. Res. Soc., vol. 54, no. 8, pp. 799-805, 2003.

[18] S. Sahoo, K. Mahesh Dash, R. C. Prusty, and A. K. Barisal, "Comparative analysis of optimal load dispatch through evolutionary algorithms,” Ain Shams Eng. J., vol. 6, no. 1, pp. 107-120, 2015. 